\title{
Testing quantum decoherence at DUNE
}

\author{
J. A. Carpio, ${ }^{1,2}$ E. Massoni, ${ }^{1}$ and A. M. Gago ${ }^{1}$ \\ ${ }^{1}$ Sección Física, Departamento de Ciencias, Pontificia Universidad Católica del Perú, \\ Apartado 1761, Lima, Perú \\ ${ }^{2}$ Department of Physics, The Pennsylvania State University, University Park, Pennsylvania 16802, USA
}

(Received 3 December 2018; revised manuscript received 22 April 2019; published 22 July 2019; corrected 22 July 2021)

\begin{abstract}
We address some theoretical issues of the quantum decoherence phenomenon within the neutrino oscillation framework and carry out various tests the under DUNE simulated experimental environment. On the theoretical side, we provide a general expression for an invariant decoherence matrix under a quantum basis rotation. On the simulated experimental side, considering a rotation-invariant and noninvariant decoherence matrix, we study the impact on the fitting of the standard oscillation parameters, the sensitivity in the mass hierarchy, and the $C P$ violation, combining the neutrino and antineutrino mode and all available neutrino oscillation probabilities channels. Furthermore, a sensitivity for the decoherence parameter of the order $10^{-24} \mathrm{GeV}$ at $3 \sigma$ is obtained for our best case. We also note that a degeneracy between the decoherence parameter and the $C P$ violation phase remains, even though our analysis includes neutrino/antineutrino mode and all probabilities channels.
\end{abstract}

DOI: 10.1103/PhysRevD.100.015035

\section{INTRODUCTION}

It is well established that neutrino oscillation is induced by a nonzero neutrino mass [1-7]. However, the existence of some, still unrevealed, subdominant mechanism is not forbidden. In general, the typical trait of this subleading effect is to the neutrino (oscillation) connection with physics beyond the Standard Model. Within this category, there are several theoretical hypotheses such as: neutrino decay [8-33], nonstandard neutrino interactions [34-40], Lorentz and $C P T$ invariance violation [41-47], etc. There is another beyond the Standard Model hypothesis which contemplates an interacting environment due to some effects produced either by strings and branes $[48,49]$ or quantum gravity [50]. The result of the interaction between the neutrino system and the environment is the introduction of decoherence/dissipative parameters into the standard oscillation framework [51,52]. Although the decoherence phenomenon applied to a neutrino system has been largely studied in the literature [51-69], there is still room for new contributions. This paper is motivated for two interesting points raised in [67]: the first was the discussion about the invariance of the decoherence matrix when we rotate the neutrino Hamiltonian in matter from the vacuum mass eigenstate basis (VMB) to the matter

Published by the American Physical Society under the terms of the Creative Commons Attribution 4.0 International license. Further distribution of this work must maintain attribution to the author(s) and the published article's title, journal citation, and DOI. Funded by SCOAP. mass eigenstates basis (MMB). The second was the possibility of having a degeneracy in oscillation probabilities between the decoherence parameter $\Gamma$ and the $C P$ violation phase $\delta$.

In this paper, we will present a detailed demonstration of the behavior of the decoherence matrix under rotations and obtain the general shape of a (rotation) invariant decoherence matrix. Furthermore, we will probe the neutrino oscillation probabilities for an invariant and noninvariant decoherence matrix under simulated experimental conditions in the context of DUNE, which will have unprecedented sensitivity to the identification of the mass hierarchy and the measurement of the $C P$ phase $\delta$ [70]. Considering the two aforementioned kinds of decoherence matrix, we will perform various tests such as: the sensitivity to the decoherence parameter and the effects of decoherence in the measurement of oscillation parameters and in the sensitivity to the mass hierarchy and $\delta$. We include, of course, the study of the degeneracy between $\Gamma$ and $\delta$, in agreement with our second motivation point.

\section{THEORETICAL CONSIDERATIONS}

\section{A. Density matrix formalism}

The description of the neutrino system weakly interacting with the environment using the open quantum system approach is given by

$$
\frac{d \hat{\rho}(t)}{d t}=-i[H, \hat{\rho}(t)]+D[\hat{\rho}(t)]
$$


the well-known Lindblad master equation, where $\hat{\rho}(t)$ is the neutrino's density matrix and $H$ is the Hamiltonian of the system. The term $D$, which is the one that encloses the dissipative/decoherence effects, is written as

$$
D[\hat{\rho}(t)]=\frac{1}{2} \sum_{j}\left(\left[\hat{\mathcal{V}}_{j}, \hat{\rho}(t) \hat{\mathcal{V}}_{j}^{\dagger}\right]+\left[\hat{\mathcal{V}}_{j} \hat{\rho}(t), \hat{\mathcal{V}}_{j}^{\dagger}\right]\right),
$$

where $\left\{\hat{\mathcal{V}}_{j}\right\}$ is a set of dissipative operators with $j=$ $1,2, \ldots, 8$ for three neutrino generations. The operators $\left\{\hat{\mathcal{V}}_{j}\right\}, \hat{\rho}$, and $H$ can be expanded in terms of the $S U(3)$ GellMann matrices and the identity matrix (although this last component is not relevant). After several intermediate manipulations, well explained in [67], we can arrive at the solution of the Lindblad master equation for a constant matter density,

$$
\vec{\rho}(t)=e^{\left(M_{H}+M_{D}\right) t} \vec{\rho}(0),
$$

where $\vec{\rho}$ is an eight-dimensional column vector consisting of the $\rho_{k}$, the components of the quoted expansion before, the $8 \times 8$ matrices $M_{H}$ and $M_{D}$, encode the Hamiltonian and decoherence components of the same expansion, respectively. The dissipative/decoherence matrix $M_{D}$, that contains all the decoherence parameters, has to be a symmetric, positive-semidefinite matrix, and its entries should satisfy a set of inequalities (see [71] for a full list).

Thus, we can get the oscillation probabilities $P\left(\nu_{\alpha} \rightarrow \nu_{\beta}\right) \equiv$ $P_{\nu_{\alpha} \nu_{\beta}}$ that can be obtained via inner products,

$$
P_{\nu_{\alpha} \nu_{\beta}}=\frac{1}{3} 1+\frac{1}{2} \vec{\rho}_{\nu_{\beta}} \cdot \vec{\rho}_{\nu_{\alpha}}(t),
$$

where $\vec{\rho}_{\nu_{\alpha}}(t)$ is the time evolved state from an initial neutrino flavor $\nu_{\alpha}$ and $\vec{\rho}_{\nu_{\beta}}$ is the final neutrino flavor $\nu_{\beta}$ to be detected. Considering that the neutrinos are ultrarelativistics, we have $t=L$, where $L$ is the baseline.

\section{B. Neutrino Hamiltonian, rotation, and $C P$ phase}

The neutrino Hamiltonian $\hat{H}_{\mathrm{VAC}}+\hat{A}$ in the $\mathrm{VMB}$ for a neutrino of energy $E_{\nu}$ is given by

$$
\begin{aligned}
H_{V}(\delta)= & \frac{1}{2 E_{\nu}}\left\{\left(\begin{array}{ccc}
0 & 0 & 0 \\
0 & \Delta \mathrm{m}_{21}^{2} & 0 \\
0 & 0 & \Delta \mathrm{m}_{31}^{2}
\end{array}\right)\right. \\
& \left.+U^{\dagger}\left(\begin{array}{ccc}
A_{\mathrm{CC}} & 0 & 0 \\
0 & 0 & 0 \\
0 & 0 & 0
\end{array}\right) U\right\},
\end{aligned}
$$

where $\quad \hat{H}_{\mathrm{VAC}}=\operatorname{Diag}\left(0, \Delta \mathrm{m}_{21}^{2} / 2 E_{\nu}, \Delta \mathrm{m}_{31}^{2} / 2 E_{\nu}\right), \quad \hat{A}=$ $U^{\dagger} \operatorname{Diag}\left(A_{\mathrm{CC}} / 2 E_{\nu}, 0,0\right) U$, with $A_{\mathrm{CC}}=2 E \sqrt{2} G_{F} n_{e}$, where
$G_{F}, n_{e}$ are the Fermi coupling constant and electron number density, respectively. The matrix $U$ is defined as

$$
U=U_{23} U_{\delta}^{\dagger} U_{13} U_{\delta} U_{12}
$$

with $U_{\delta}=\operatorname{Diag}\left(1,1, e^{-i \delta}\right)$. Considering that the matrix $U_{23} U_{\delta}^{\dagger}$ commutes with $\operatorname{Diag}\left(A_{\mathrm{CC}}, 0,0\right)$ and the matrix $U_{\delta}$ commutes with $U_{12}$ and $\operatorname{Diag}\left(0, \Delta \mathrm{m}_{21}^{2}, \Delta \mathrm{m}_{31}^{2}\right)$, separately, the neutrino Hamiltonian can be rewritten as follows:

$$
H_{V}(\delta)=U_{\delta}^{\dagger} H_{V}(0) U_{\delta},
$$

where $H_{V}(0)$ is given by

$$
\begin{aligned}
H_{V}(0)= & \frac{1}{2 E_{\nu}}\left\{\left(\begin{array}{ccc}
0 & 0 & 0 \\
0 & \Delta \mathrm{m}_{21}^{2} & 0 \\
0 & 0 & \Delta \mathrm{m}_{31}^{2}
\end{array}\right)\right. \\
& \left.+U_{12}^{\dagger} U_{13}^{\dagger}\left(\begin{array}{ccc}
A_{\mathrm{CC}} & 0 & 0 \\
0 & 0 & 0 \\
0 & 0 & 0
\end{array}\right) U_{13} U_{12}\right\} .
\end{aligned}
$$

The Hamiltonian $H_{V}$ defined in the VMB can be related with the Hamiltonian $\hat{H}_{M}$ in the MMB (this is where the Hamiltonian is diagonal) through

$$
\hat{H}_{M}(\delta)=U_{T}^{\dagger}\left(\delta, \phi_{1}, \phi_{2}\right) H_{V}(\delta) U_{T}\left(\delta, \phi_{1}, \phi_{2}\right) .
$$

Using Eq. (7) and that $H_{M}(\delta)$ commutes with the unitary phase operator $U_{\phi}^{\dagger}\left(\phi_{1}, \phi_{2}\right)=\operatorname{Diag}\left(1, e^{-i \phi_{1}}, e^{-i \phi_{2}}\right)$ being $\phi_{1}$ and $\phi_{2}$ arbitrary phases, the latter equation can be rewritten as follows:

$$
\begin{aligned}
H_{M}(\delta)= & U_{\phi}^{\dagger}\left(\phi_{1}, \phi_{2}\right) U_{T}^{\dagger}\left(\delta, \phi_{1}, \phi_{2}\right) U_{\delta}^{\dagger} H_{V}(0) \\
& \times U_{\delta} U_{T}\left(\delta, \phi_{1}, \phi_{2}\right) U_{\phi}\left(\phi_{1}, \phi_{2}\right) .
\end{aligned}
$$

Given that $H_{M}(\delta)$ is represented by a unitary transformation of the operator $H_{V}(0)$, the eigenvalues of the former do not depend on $\delta$ or the phases $\phi_{1}, \phi_{2}$. We thus write

$$
H_{M}(\delta)=H_{M}(0)=U_{T}^{\dagger}(0,0,0) H_{V}(0) U_{T}(0,0,0) .
$$

Comparing Eqs. (10) and (11), we obtain

$$
U_{T}\left(\delta, \phi_{1}, \phi_{2}\right)=U_{\delta}^{\dagger} U_{T}(0,0,0) U_{\phi}^{\dagger}\left(\phi_{1}, \phi_{2}\right) .
$$

This result implies that once we find a matrix $U_{T}(0,0,0)$ that diagonalizes $H_{V}(0)$, a general $U_{T}\left(\delta, \phi_{1}, \phi_{2}\right)$ that diagonalizes $H_{V}(\delta)$ can be constructed. 


\section{Invariant matrices analysis}

As mentioned in Ref. [67], the relationship between the $\mathrm{VMB}$ and MMB decoherence matrices goes as follows:

$$
M_{D}^{M}\left(\delta, \phi_{1}, \phi_{2}\right)=P\left(\delta, \phi_{1}, \phi_{2}\right) M_{D}^{V} P^{T}\left(\delta, \phi_{1}, \phi_{2}\right) .
$$

The matrix $P\left(\delta, \phi_{1}, \phi_{2}\right)$ is defined as

$$
P\left(\delta, \phi_{1}, \phi_{2}\right)=F\left(\phi_{1}, \phi_{2}\right) P_{0} R(\delta),
$$

where further details about $P_{0}=P(0,0,0), R(\delta)$ and $F\left(\phi_{1}, \phi_{2}\right)$ are given in the Appendix B. Although we are emphasizing the dependence of $M_{D}^{M}$ on $\delta$ and the arbitrary phases $\phi_{1}$ and $\phi_{2}$ in the Eq. (13), it is important to notice that this matrix, in general, may also depend on the neutrino energy and the matter potential $A$.

We rewrite Eq. (13) more explicitly,

$$
\begin{aligned}
M_{D}^{M}\left(\delta, \phi_{1}, \phi_{2}\right)= & F\left(\phi_{1}, \phi_{2}\right) P_{0} R(\delta) M_{D}^{V} \\
& \times R^{T}(\delta) P_{0}^{T} F^{T}\left(\phi_{1}, \phi_{2}\right),
\end{aligned}
$$

and wish to impose the condition $M_{D}^{M}=M_{D}^{V}$, meaning that the decoherence matrix $M_{D}^{V}$ is invariant under rotations in $P$. This situation is very convenient to deal with since the neutrino matter oscillation plus quantum decoherence probability formulas are easily found from its corresponding formulas in vacuum (see [67]).

We want to prove that, given an invariant matrix $M_{D}^{V}$ when $\delta=0$, it is possible to derive invariant matrices $\bar{M}_{D}^{V}$ for other $\delta$. We start with the invariance condition $M_{D}^{M}\left(\delta, \phi_{1}, \phi_{2}\right)=M_{D}^{V}$ when $\delta=0$,

$$
M_{D}^{V}=F\left(\phi_{1}, \phi_{2}\right) P_{0} M_{D}^{V} P_{0}^{T} F^{T}\left(\phi_{1}, \phi_{2}\right) .
$$

For the case $\delta \neq 0$, the invariance condition for $M_{D}^{M}\left(\delta, \tilde{\phi}_{1}, \tilde{\phi}_{2}\right)=\bar{M}_{D}^{V}$ reads

$$
\begin{aligned}
\bar{M}_{D}^{V}= & F\left(\tilde{\phi}_{1}, \tilde{\phi}_{2}\right) P_{0} R(\delta) \bar{M}_{D}^{V} \\
& \times R^{T}(\delta) P_{0}^{T} F^{T}\left(\tilde{\phi}_{1}, \tilde{\phi}_{2}\right) .
\end{aligned}
$$

Imposing the following relation to the preceding equation:

$$
\bar{M}_{D}^{V}=R^{T}(\delta) M_{D}^{V} R(\delta),
$$

together with Eq. (16), we get

$$
\begin{aligned}
M_{D}^{M}\left(\delta, \tilde{\phi}_{1}, \tilde{\phi}_{2}\right)= & F\left(\tilde{\phi}_{1}, \tilde{\phi}_{2}\right) F^{T}\left(\phi_{1}, \phi_{2}\right) M_{D}^{V} \\
& \times F\left(\phi_{1}, \phi_{2}\right) F^{T}\left(\tilde{\phi}_{1}, \tilde{\phi}_{2}\right) .
\end{aligned}
$$

Thus, if we demand $F\left(\phi_{1}, \phi_{2}\right) F^{T}\left(\tilde{\phi}_{1}, \tilde{\phi}_{2}\right)=R(\delta)$, the condition $M_{D}^{M}\left(\delta, \tilde{\phi}_{1}, \tilde{\phi}_{2}\right)=\bar{M}_{D}^{V}$ is achieved, and $\bar{M}_{D}^{V}$ is invariant.
In Ref. [67], it is found that for $\delta=0, \pi$ the invariant matrix $M_{D}^{V}$ is given by

$$
M_{D}^{V}=-\operatorname{Diag}\left(\Gamma_{1}, \Gamma_{2}, \Gamma_{1}, \Gamma_{1}, \Gamma_{2}, \Gamma_{1}, \Gamma_{2}, \Gamma_{1}\right),
$$

with $\Gamma_{1} / 3 \leq \Gamma_{2} \leq 5 \Gamma_{1} / 3$. Substituting this $M_{D}^{V}$ into Eq. (18), we can extrapolate that the corresponding invariant $\hat{M}_{D}^{V}$ for any $\delta$ is described by the following block diagonal matrix:

$$
\bar{M}_{D}^{V}=R^{T}(\delta) M_{D}^{V} R(\delta)=-\left(\begin{array}{cccccc}
\Gamma_{1} & & & & & \\
& \Gamma_{2} & & & & \\
& & \Gamma_{1} & & & \\
& & & Q & & \\
& & & & Q & \\
& & & & & \Gamma_{1}
\end{array}\right) \text {, }
$$

where the matrix $Q$ is

$$
Q=\left(\begin{array}{cc}
\Gamma_{1} \cos ^{2} \delta+\Gamma_{2} \sin ^{2} \delta & \left(\Gamma_{1}-\Gamma_{2}\right) \cos \delta \sin \delta \\
\left(\Gamma_{1}-\Gamma_{2}\right) \cos \delta \sin \delta & \Gamma_{2} \cos ^{2} \delta+\Gamma_{1} \sin ^{2} \delta
\end{array}\right) .
$$

From the latter equation, we can calculate $\hat{M}_{D}^{V}$ for $\delta=\pi / 2,3 \pi / 2$ getting

$$
\begin{aligned}
\bar{M}_{D}^{V} & =R^{T}(\delta) M_{D}^{V} R(\delta) \\
& =-\operatorname{Diag}\left(\Gamma_{1}, \Gamma_{2}, \Gamma_{1}, \Gamma_{2}, \Gamma_{1}, \Gamma_{2}, \Gamma_{1}, \Gamma_{1}\right),
\end{aligned}
$$

similar to the one mentioned in Ref. [67].

\section{Adding Hamiltonian terms in the weak coupling limit}

At this point, it is interesting to make a comparison between the approach given in [68] for defining invariant matrices, and the one presented here and in [67]. With the purpose of demanding energy conservation, the following conditions are imposed in [68]: $\left[H_{\mathrm{VAC}}, \hat{\mathcal{V}}_{j}\right]=0$ and $\left[\hat{H}_{M}, \hat{\mathcal{V}}_{j}^{M}\right]=0$, being that $\hat{\mathcal{V}}_{j}$ and $\hat{\mathcal{V}}_{j}^{M}$ are the dissipator operators written in the VMB (for the pure vacuum case) and in the MMB, respectively. To achieve the aforementioned conditions, it is required that $\hat{\mathcal{V}}_{j}$ changes when the matter potential term $\hat{A}$ is added to the vacuum Hamiltonian $H_{\mathrm{VAC}}$, all of this in the VMB. The new dissipator operator is defined by $\hat{\mathcal{V}}_{j}^{\text {new }}=U_{T} \hat{\mathcal{V}}_{j} U_{T}^{\dagger}$, depending on the matter potential and the neutrino energy, in a way that it recovers its vacuum form when it is rotated to the MMB; this is $\hat{\mathcal{V}}_{j}^{M}=U_{T}^{\dagger} \hat{\mathcal{V}}_{j}^{\text {new }} U_{T}=U_{T}^{\dagger} U_{T} \hat{\mathcal{V}}_{j} U_{T}^{\dagger} U_{T}=\hat{\mathcal{V}}_{j}$. This situation is incongruent with the underlying regime used for describing the neutrinos as an open quantum system, which is the 
Born-Markov approximation (BM). The Born approximation is applied when the environment interacts weakly with the neutrino system [72,73], while the Markovianity assumption implies the use of the Lindblad form [Eq. (2)] [74,75]. In this evolution, the system is modified by the environment and parametrized by the dissipator operators (i.e., the decoherence matrix), but not viceversa. Thereby, the environment is considered unaffected by the system, and the addition of the matter potential term to the vacuum neutrino Hamiltonian should not change (or in a negligible way) the dissipator operator $\hat{\mathcal{V}}_{j}$.

\section{E. Selected decoherence matrix models}

Our analysis will be limited to diagonal decoherence matrices due to their relative simplicity. While analytical expressions are available for these matrices in the context of vacuum oscillations [53], oscillations in matter can be found through perturbative expansions. For a generic matrix $M_{D}^{V}=-\operatorname{Diag}\left(\Gamma_{1}, \Gamma_{2}, \Gamma_{3}, \Gamma_{4}, \Gamma_{5}, \Gamma_{6}, \Gamma_{7}, \Gamma_{8}\right)$, we can make an expansion in the small quantities $\alpha=\Delta m_{21}^{2} /$ $\Delta m_{31}^{2}, \theta_{13}$ and $\bar{\Gamma}_{i}=\Gamma_{i} L$, assuming the latter is $<0.1$. The lowest order contribution to the transition probability $P_{\nu_{\mu} \nu_{e}}$ is found to be

$$
\begin{aligned}
P_{\nu_{\mu} \nu_{e}}= & P_{\nu_{\mu} \nu_{e}}^{(0)}+\frac{1}{4} \cos ^{2} \theta_{23}\left[\bar{\Gamma}_{1}+\bar{\Gamma}_{3}+\cos 4 \theta_{12}\left(\bar{\Gamma}_{3}-\bar{\Gamma}_{1}\right)\right] \\
& +\frac{1}{12}\left(1-3 \cos 2 \theta_{23}\right) \bar{\Gamma}_{8}
\end{aligned}
$$

where $P_{\nu_{\mu} \nu_{e}}^{(0)}$ is the standard oscillation transition probability. We also notice that only three decoherence parameters of such a matrix dominate the contributions to the probability. Furthermore, these terms do not depend on the neutrino energy or matter potential, leading to a probability shift across all energies - a property that will help us qualitatively explain our results. Using the current values of the standard oscillation (SO) parameters given in Table I, this shift turns out to be positive.

In the $\nu_{\mu} \rightarrow \nu_{\mu}$ channel, decoherence turns out to be a subdominant effect and does not significantly affect the shape of the curves, while in the $\nu_{\mu} \rightarrow \nu_{e}$ channel, it is the

TABLE I. Relevant parameters assumed for our DUNE analysis.

\begin{tabular}{lcc}
\hline \hline Parameter & Value & Error \\
\hline$\theta_{12}$ & $33.62^{\circ}$ & $0.77^{\circ}$ \\
$\theta_{23}$ & $47.2^{\circ}$ & $2.9^{\circ}$ \\
$\theta_{13}$ & $8.54^{\circ}$ & $0.15^{\circ}$ \\
$\frac{\Delta m_{21}^{2}}{10^{-5} \mathrm{eV}^{2}}$ & 7.40 & 0.21 \\
$\frac{\Delta m_{31}^{2}}{10^{-3} \mathrm{eV}^{2}}$ & 2.494 & 0.032 \\
$\delta$ & Varies & $\cdots$ \\
$\rho$ & $2.97 \mathrm{~g} \mathrm{~cm}^{-3}$ & $\cdots$ \\
Baseline $L$ & $1300 \mathrm{~km}$ & $\cdots$ \\
\hline \hline
\end{tabular}

leading term (the standard oscillation (SO) transition probability is led by terms proportional to $\theta_{13}^{2}, \alpha^{2}$ and $\left.\alpha \theta_{13}\right)$. For that reason we are not showing these probabilities, although this channel is included in our analysis.

\section{Model A}

In light of this, the first one-parameter model we propose for this study (referred from now on as model A) assumes $\Gamma_{3}=\Gamma_{8}=0$,

$$
M_{D}^{V}=-\operatorname{Diag}(\Gamma, \Gamma, 0, \Gamma / 4, \Gamma / 4, \Gamma / 4, \Gamma / 4,0),
$$

which is an noninvariant decoherence matrix. The approximate expression for the transition probability $\left(\nu_{\mu} \rightarrow \nu_{e}\right)$ is

$$
\begin{aligned}
P_{\nu_{\mu} \nu_{e}}= & P_{\nu_{\mu} \nu_{e}}^{(0)}+\frac{1}{2} \bar{\Gamma} \cos ^{2} \theta_{23} \sin ^{2} 2 \theta_{12} \\
& -\frac{1}{4} \cos ^{2} \theta_{23} \bar{\Gamma}^{2}\left[\sin ^{4} 2 \theta_{12}+\sin ^{2} 4 \theta_{12} \frac{\sin ^{2}(A \Delta)}{4 A^{2} \Delta^{2}}\right] \\
& +\frac{\bar{\Gamma} \theta_{13} \sin 2 \theta_{23} \sin 4 \theta_{12}}{4(1-A) A \Delta}[\sin (A \Delta) \cos (\delta+A \Delta) \\
& \left.-A^{2} \sin \Delta \cos (\delta+\Delta)\right] \\
& -\frac{\alpha \bar{\Gamma}}{2 A^{2} \Delta} \cos 2 \theta_{12} \cos ^{2} \theta_{23} \sin ^{2} 2 \theta_{12} \times(\sin 2 A \Delta-2 A \Delta) .
\end{aligned}
$$

In this expression, $\bar{\Gamma}=\Gamma L, \Delta=\Delta m_{31}^{2} L / 4 E$ and $A=$ $A_{\mathrm{CC}} / \Delta m_{31}^{2}$. To obtain the antineutrino oscillation probabilities, switch $A \rightarrow-A$ and $\delta \rightarrow-\delta$.

\section{Model B}

The second model, from now on named model $\mathrm{B}$, is an invariant matrix based on (24),

$$
M_{D}^{V}=-\operatorname{Diag}(\Gamma, 5 \Gamma / 3, \Gamma, \Gamma, 5 \Gamma / 3, \Gamma, 5 \Gamma / 3, \Gamma) .
$$

For such a matrix, we do not have a transition probability formula akin to (27). However, we may still use (25) to get a qualitative understanding of our results referred to this model.

\section{Analytical comparison between model $A$ and $B$}

The neutrino and antineutrino transition probabilities are displayed in Fig. 1 for both of our proposed decoherence models. As expected from the perturbative formula, the main feature is the increased transition probability at all energies. Note that the rise in the oscillation probability is greatest for model $\mathrm{B}$, consistent with (25) now that $\Gamma_{8} \neq 0$ increasing the value of the shift. The peaks in the transition probability occur between 2 and $4 \mathrm{GeV}$ for both channels. 


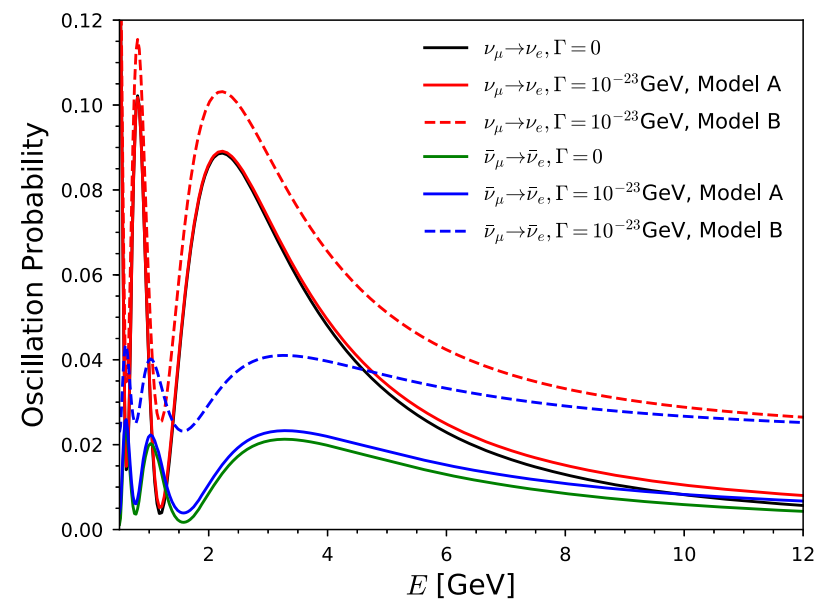

FIG. 1. Neutrino and antineutrino transition probabilities for the decoherence models discussed in Sec. II E for decoherence parameters $\Gamma=0,10^{-23} \mathrm{GeV}$. The value $\delta=-\pi / 2$ is assumed.

\section{SIMULATION AND RESULTS}

For DUNE simulations, we will use the optimized beam as described in the Conceptual Design Report [70] and make use of the files from Ref. [76]. We use an exposure of $150 \mathrm{kt}$ MW year exposure each for the neutrino and antineutrino channels. Both the neutrino mode (forward horn currentFHC) and antineutrino mode (reverse horn current-RHC) are used for 3.5 yrs on each. We are also using both the appearance $\left(\nu_{\mu} \rightarrow \nu_{e}\right)$ and disappearance $\left(\nu_{\mu} \rightarrow \nu_{\mu}\right)$ channels on neutrino and antineutrino modes. All neutral- and charged-current interaction background rates discussed in detail in Ref. [70] Sec. III.6.1 are included.

Throughout this section, we use the SO parameters from the global fit based on data available in January 2018 $[77,78]$, assuming normal ordering (NO) and some modifications. The ranges provided are not symmetric about the fit values, but we will take them as symmetric by taking the average of the one-sided deviations. Due to the relatively unconstrained value of $\delta$, we consider different values for this parameter with special emphasis on $\delta=-\pi / 2$, which is rather similar to the current best fit value. As such, no priors are assigned to $\delta$. We summarize this information in Table I.

For all subsequent statistical analyses, we will use the GLoBES package $[79,80]$, while oscillation probabilities are calculated using NuSQuIDS [81].

This study relies on the $\chi^{2}$ test statistic to compare data generated by a set of "true" parameters $\xi^{\text {true }}$ against a hypothesis that assumes a set of "test" values $\xi$. Unless otherwise stated, the parameters $\xi^{\text {true }}$ will have the values in Table I. The values of $\delta$ and $\Gamma$ will be provided on a caseby-case basis. In the context of DUNE and GLoBES, $\chi^{2}$ is defined as

$$
\chi^{2}\left(\xi, \xi^{\text {true }}\right)=\sum_{i} \frac{\left(N_{i}(\xi)-N_{i}\left(\xi^{\text {true }}\right)\right)^{2}}{N_{i}\left(\xi^{\text {true }}\right)}
$$

where the $N_{i}$ is the expected number of events in the $i$ 'th energy bin. When including priors, GLoBES modifies $\chi^{2}$ by adding an extra term,

$$
\chi^{2} \rightarrow \chi^{2}+\sum_{j} \frac{\left(\xi_{j}-\xi_{j}^{\text {true }}\right)^{2}}{\sigma_{j}^{2}},
$$

where the summation in $j$ is over all parameters for which $\sigma_{j} \neq 0$. In this and all subsequent definitions for $\chi^{2}$, it is implicit that $\Gamma$ and $\gamma$ are used interchangeably depending on the assumed model.

\section{A. Determination of standard oscillation parameters}

Our first test consists of performing (Standard Oscillation) SO fits on data that include decoherence. The data are generated assuming the true parameter values in Table I, for $\delta^{\text {true }}=-\pi / 2$ and $\Gamma^{\text {true }}$ and no priors. The test hypothesis is $\Gamma=0$, and we obtain $\chi_{\min }^{2}$ by marginalizing over the SO parameters, attaining its minimum for a set of parameters which we label as "fit". We project the $\chi^{2}$ function on the $\theta_{13}-\delta$ plane,

$$
\begin{aligned}
\Delta \chi^{2}= & \chi^{2}\left(\theta_{13}, \delta, \Gamma=0, \delta^{\text {true }}, \Gamma^{\text {true }}\right) \\
& -\chi_{\min }^{2}\left(\theta_{13}^{\text {fit }}, \delta^{\text {fit }}, \Gamma=0, \delta^{\text {true }}, \Gamma^{\text {true }}\right),
\end{aligned}
$$

where all unmentioned test parameters are fixed to their fit values.

We plot our results in Fig. 2. As we increase the value of $\Gamma^{\text {true }}$, the SO fit shifts towards higher values of $\theta_{13}$ for both models. We can explain this behavior through Eq. (25), where as long as we increase $\Gamma$, the $\nu_{\mu} \rightarrow \nu_{e}$ transition probability grows in an energy-independent way, which, if it is fitted under the $\mathrm{SO}$ assumption, can be misconstrued as a larger mixing angle $\theta_{13}$. Both plots have similar shapes, where model $\mathrm{B}$ has a more notable shift to higher $\theta_{13}$, a feature that is explained by looking at the transition probabilities in Fig. 1, with higher shifts appearing due to the nonzero $\Gamma_{3}, \Gamma_{8}$ [see Eq. (25)]. As expected, the more serious discrepancy between model $\mathrm{B}$ and $\mathrm{SO}$, in comparison with the corresponding between model A and SO, is also translated in a more relevant quantity, which is the event rate (see Appendix C). It is important to note that this higher sensitivity of model $\mathrm{B}$ will be reflected in all the successive analysis (plots) presented in this manuscript.

On the other hand, the general shape of the contour plots remains the same. We also made the same analysis using the FHC and RHC separately, where the former provided stronger constraints compared to the latter, because of the higher statistics of the former. Similarly, we found the disappearance probability alone does not provide good restrictions on the parameter space based on the small effect decoherence has on the $\nu_{\mu} \rightarrow \nu_{\mu}$ probability. We thus expect the largest contributions to $\Delta \chi^{2}$ to come from the appearance channel. 

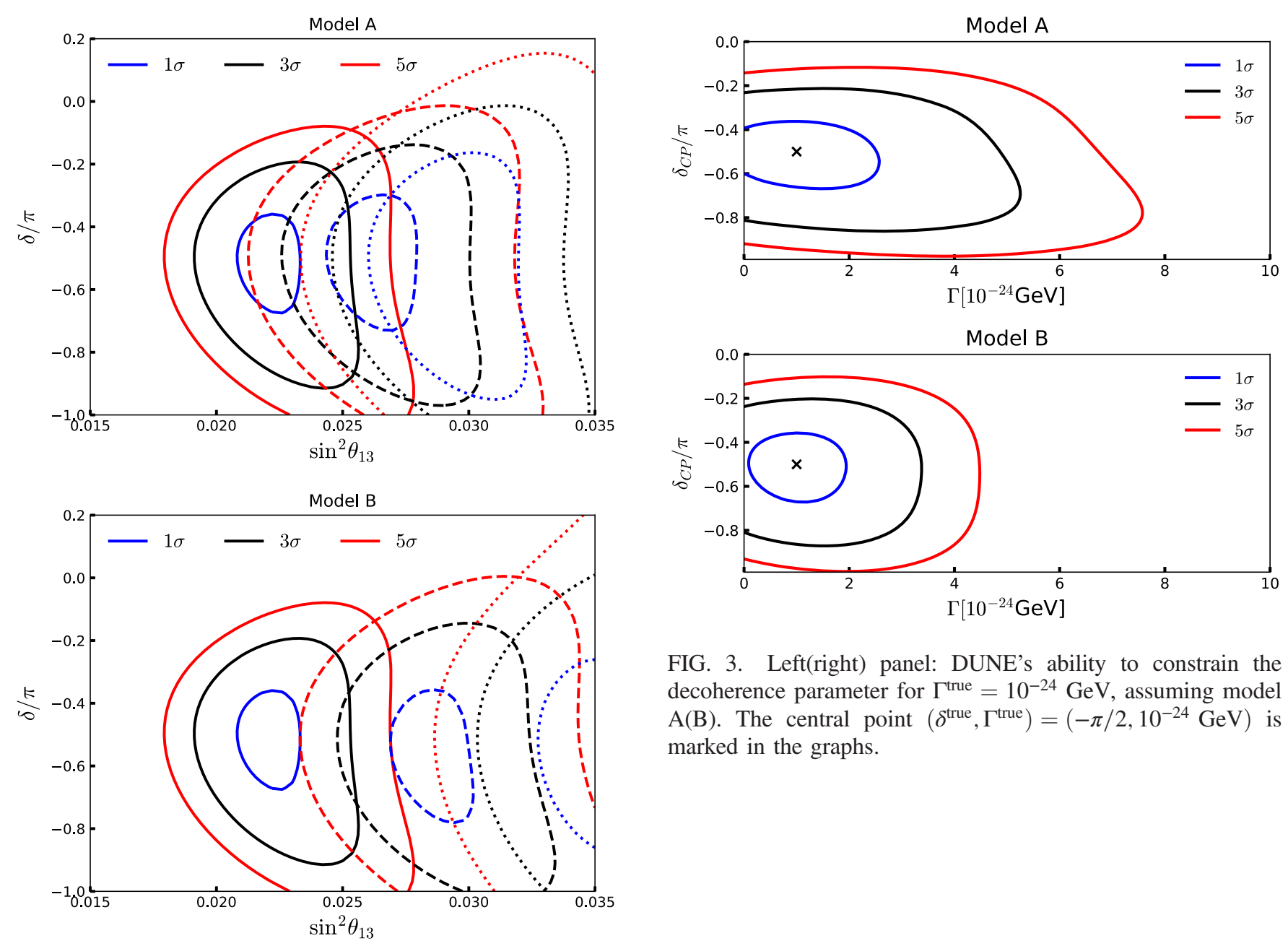

FIG. 3. Left(right) panel: DUNE's ability to constrain the decoherence parameter for $\Gamma^{\text {true }}=10^{-24} \mathrm{GeV}$, assuming model $\mathrm{A}(\mathrm{B})$. The central point $\left(\delta^{\text {true }}, \Gamma^{\text {true }}\right)=\left(-\pi / 2,10^{-24} \mathrm{GeV}\right)$ is marked in the graphs.

FIG. 2. Effects of decoherence on standard oscillation fits for $\delta^{\text {true }}=-\pi / 2$. We show the $\Delta \chi^{2}$ contours or $\Gamma^{\text {true }}=0 \mathrm{GeV}$, $2 \times 10^{-24} \mathrm{GeV}$, and $5 \times 10^{-24} \mathrm{GeV}$ (solid, dashed, and dotted lines, respectively).

We point out that short baseline neutrino oscillation experiments can measure the value of $\theta_{13}$ quite precisely and will not be confused with the $\mathrm{SO}+$ decoherence scenario, since $\Gamma L$ is much smaller than in DUNE. The precision on $\theta_{13}$ is also expected to improve by the time DUNE becomes operational. An interesting possibility arises: if DUNE's measurement of $\theta_{13}$ is incompatible with those obtained from reactor experiments, then decoherence would provide an explanation for this discrepancy, particularly if DUNE's measured $\theta_{13}$ is larger than the accepted value.

\section{B. Effects of decoherence on constraining $\delta$}

To test the ability of DUNE to constrain decoherence parameters, we generate data assuming true values for $\delta$ and $\Gamma$ and make a $\chi^{2}$ plot on the $\delta, \Gamma$ plane. Our $\chi^{2}$ in this case assumes $\delta$ and $\Gamma$ as free parameters, and all remaining parameters are fixed to their true fit values (there is no marginalization here). Our findings are displayed in Fig. 3.

If we assume $\Gamma^{\text {true }}=10^{-24} \mathrm{GeV}$, we find that the data are still compatible with $\Gamma / 10^{24} \mathrm{GeV}=2.3,5.1,7.4(1.8$, 3.3 , and 4.3) for model A (B) at 1,3 , and $5 \sigma$, respectively. We note that model B's limits are more stringent than model A; a feature explained mostly by the increased contribution from the first order correction to the transition probability [see Eq. (25)]. For this assumed value of $\Gamma^{\text {true }}$, both models are able to exclude the standard oscillation scenario at the $1 \sigma$ level.

Even in the presence of decoherence, $\delta$ is well constrained. In [67], we pointed out that the $C P$ assymmetries for $\delta= \pm \pi / 2$ were similar for low neutrino energies, and it was possible to confuse these two values of $\delta$ in the presence of decoherence. We also mentioned that after taking the whole of DUNE's energy range into account there was no similarity remaining. The aforementioned point is consistent with our current results, where we found that our plots have no secondary contours appearing in the vicinity of $\delta=\pi / 2$.

\section{Constraining the decoherence parameter}

For this study, the simulated data assume $\Gamma^{\text {true }}=0$, and a fit is performed for a given test parameter $\Gamma$. Our test statistic is 


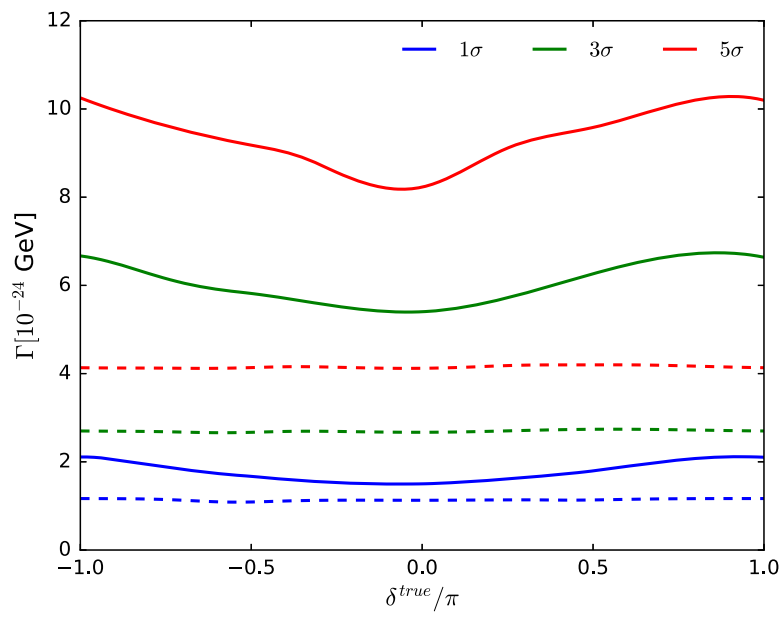

FIG. 4. Sensitivity to the decoherence parameter $\Gamma$, as a function of $\delta^{\text {true }}$, for different confidence levels. Solid lines correspond to model A, and dashed lines correspond to model B.

$$
\chi^{2}(\Gamma)=\chi^{2}\left(\Gamma, \delta^{\text {true }}, \Gamma^{\text {true }}=0\right),
$$

where we marginalize over all standard oscillation parameters. We show the results in Fig. 4. The sensitivity to $\Gamma /\left(10^{-24} \mathrm{GeV}\right)$ for the model $\mathrm{A}$ is in the range 1.5-2.1, 5.4-6.7, and 8.2-10.3 at the $1 \sigma, 3 \sigma$, and $5 \sigma$ levels. On the other hand, the mode of the invariant matrix, model B, has a sensitivity almost invariant in $\delta$, and it is stronger than one predicted for model A, as we have already seen it in Fig. 3. This is a result of the increased contribution from the first order decoherence term for model B (see Fig. 1), which does not depend on the $C P$ phase; the effect of other $\delta$-dependent terms in a perturbative expansion are suppressed, such as the $\Gamma \theta_{13}$ term present in Eq. (27).
For either model, DUNE's sensitivity to $\Gamma$ is superior to the previously reported KamLAND's 95\% C.L. sensitivity of $6.8 \times 10^{-22} \mathrm{GeV}$ [56] and the $90 \%$ C.L. sensitivity of $1.2 \times 10^{-23} \mathrm{GeV}$ [68]. Our current results are also of the same order of magnitude as those reported in a recent IceCube study [69]. We do remark that the decoherence models used in these previous studies are slightly different than ours and act as benchmarks.

\section{Mass ordering and $\boldsymbol{C P}$ violation sensitivity}

If decoherence is present in nature, it is possible in principle that $\mathrm{SO}$ fits become affected due to an attempt to fit data to an inadequate hypothesis. In particular, an SO fit to the decoherence data may identify $C P$ violation and wrongfully associate it with a nonzero $\delta$. A similar situation could arise in the case of mass ordering, where DUNE's ability to discriminate between normal and inverted orderings (IO).

Mass ordering sensitivity is obtained by comparing generated data from anNO assumption with decoherence against an IO hypothesis without decoherence. When generating data, a value of $\Gamma^{\text {true }}$ is assumed and the test statistic is marginalized in all oscillation parameters while keeping $\Gamma=0$ fixed, meaning that

$$
\chi_{\mathrm{MO}}^{2}=\chi^{2}\left(\Delta m_{31}^{2}<0, \Gamma=0, \Delta m_{31}^{2 \text { true }}>0, \delta^{\text {true }}, \Gamma^{\text {true }}\right) .
$$

The sensitivities are presented in Fig. 5 for different $\Gamma^{\text {true }}$. We see that, regardless of $\delta^{\text {true }}$ and our assumed decoherence model, the sensitivity is well above $5 \sigma$ for our chosen values of the decoherence parameter and in fact improves it. To explain this feature, we point out that the
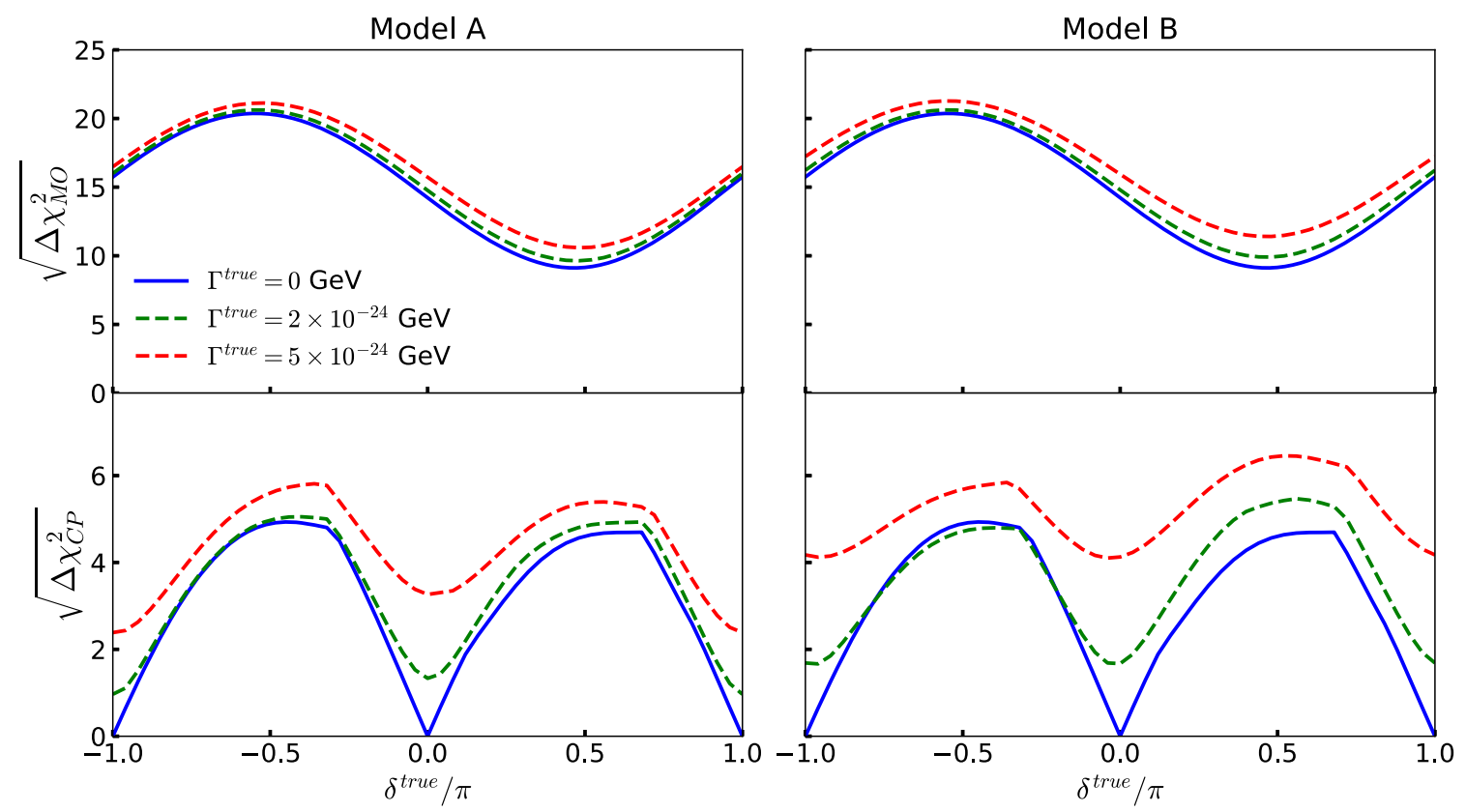

FIG. 5. Mass ordering and $C P$ violation sensitivity for our decoherence matrix models, assuming a standard oscillation fit hypothesis. 

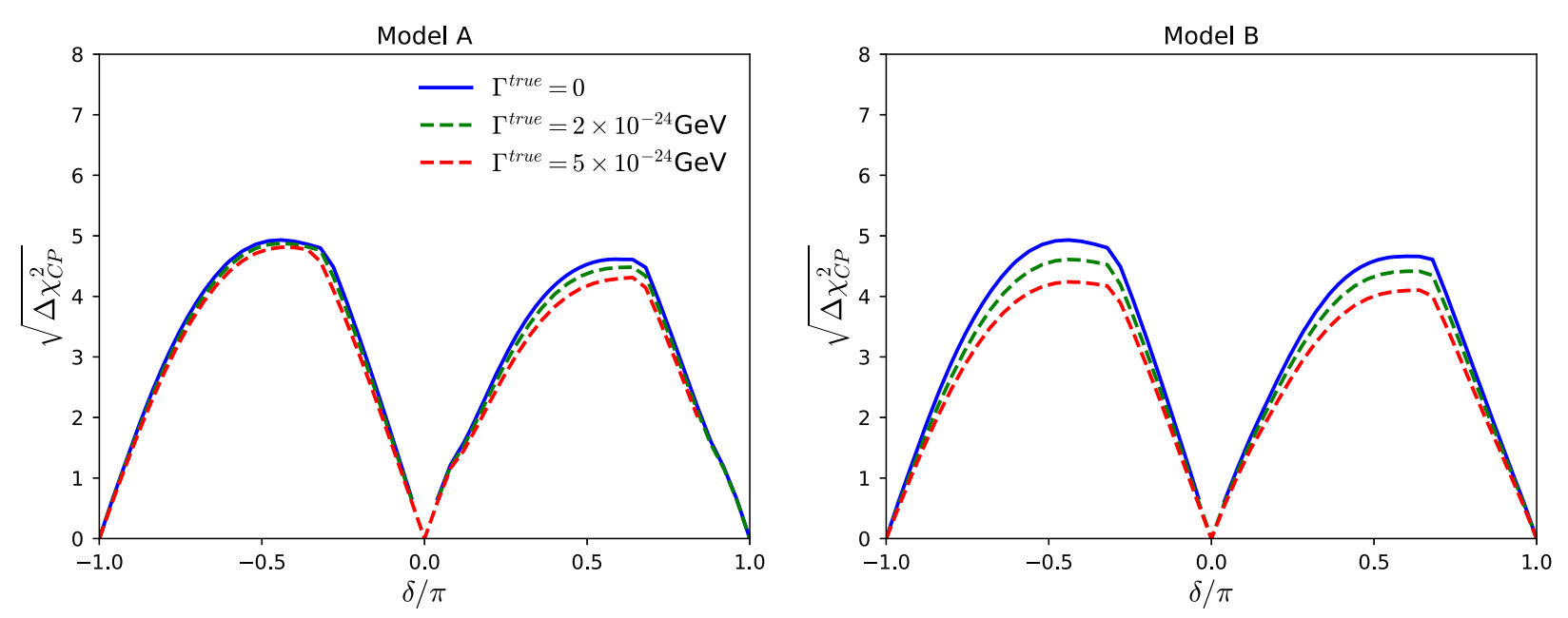

FIG. 6. Same as the top panels in Fig. 5, but the fit hypothesis assumes decoherence.
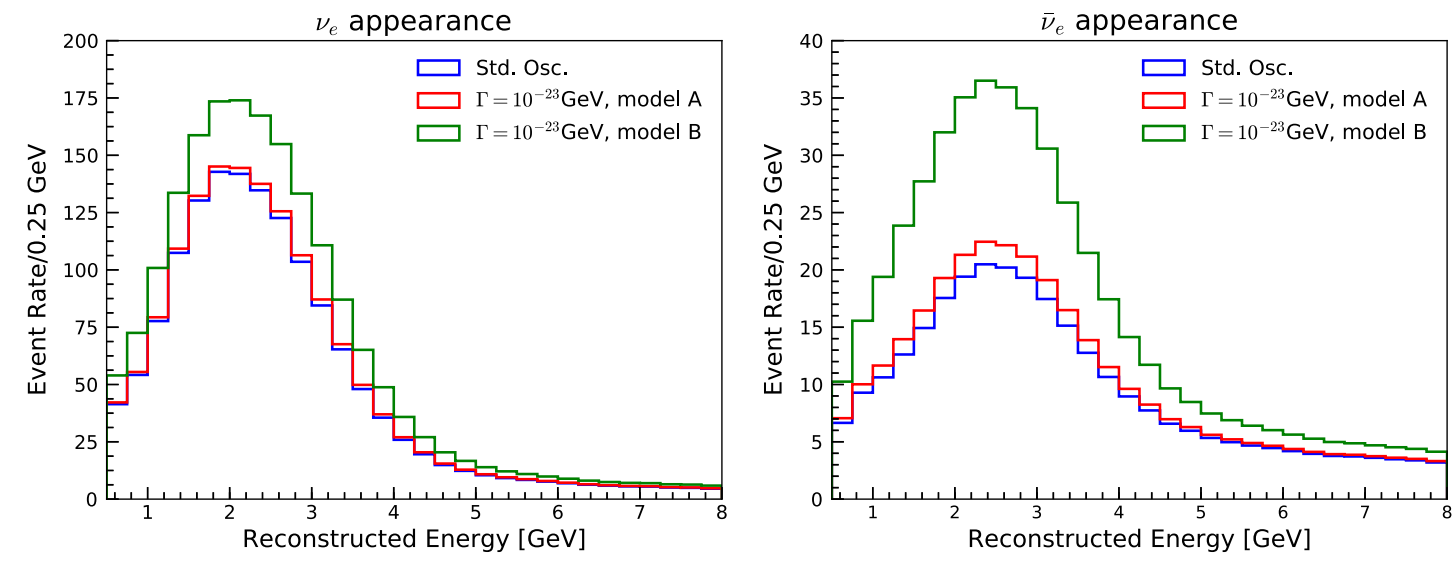

FIG. 7. Total (signal + background) appearance event rates for our decoherence models, assuming $\delta=-\pi / 2$. Neutrino (antineutrino) channel is shown in the left (right) panel.

IO fit yielded similar values for the SO parameters for all $\delta^{\text {true }}$, with the fit value $\delta^{\text {it }}$ in the vicinity of $\delta=-\pi / 2$. This occurred for all the $\Gamma^{\text {true }}$ that we analyzed. When we studied the IO fit, we noticed that the probability in the $\nu_{\mu} \rightarrow \nu_{e}$ channel is lower, and more well separated, than that corresponding to the NO data in the energy range 2-4 GeV. Being that, as long as $\Gamma^{\text {true }}$ increases, the NO transition probabilities do as well. In return, the resulting IO fit transition probabilities separate more and more from the corresponding NO assumptions from the data. This separation leads to the observed increase in sensitivity. The impact of $\Gamma$ on the sensitivity is strongest as we approach $\delta^{\text {true }}=\pi / 2$.

To obtain DUNE's sensitivity to $C P$ violation, we adopt a definition similar to the one presented in [70],

$$
\begin{gathered}
\chi_{C P}^{2}=\min \left[\chi^{2}\left(\delta=0, \Gamma=0, \delta^{\text {true }}, \Gamma^{\text {true }}\right),\right. \\
\left.\chi^{2}\left(\delta=\pi, \Gamma=0, \delta^{\text {true }}, \Gamma^{\text {true }}\right)\right]
\end{gathered}
$$

and we marginalize over all unmentioned test parameters. We checked that, after marginalization, the oscillation parameters remain approximately the same, with the exception of $\theta_{13}$ that settles at higher values to account for the increased event rates in the appearance channels due to decoherence. The IO alternative was never preferred during the minimization procedure of $\chi^{2}$. This observation is easily explained by Fig. 5 because DUNE can distinguish between the hierarchies with a large significance.

The data generated using $\mathrm{NO}$ have an additional fake $C P$ violation contribution coming from the matter potential present in the term proportional to $\Gamma \theta_{13}$, which is mistaken in an $\mathrm{SO}$ fit as a nonzero $C P$ violation. For this reason, even when $\delta^{\text {true }}=0, \pi$, decoherence may cause us to reject the null hypothesis (no $C P$ violation). Due to this decoherence induced $C P$ violation, decoherence lets us reject the null hypothesis at a higher confidence level throughout all values of $\delta$. The aforementioned case is useful since this illustrates the consequences of disregarding decoherence in the theoretical hypothesis, despite its presence in nature (the data). 
An alternative case is to redefine Eqs. (33) and (34) so that $\Gamma$ becomes a free parameter (i.e., we assume decoherence in both the generated data and the fit hypothesis). When this happens, we find that sensitivity decreases both in $C P$ and mass ordering, and the results are shown in Fig. 6. It is natural that $\chi^{2}$ decreases once we release the constraint on $\Gamma$, as it expands the parameter space that can be explored during minimization. In particular, decoherence introduces additional $C P$ violation effects which are present even when we assume $\delta=0$ in our hypothesis, allowing for improved fits and lowers $\Delta \chi^{2}$. Interestingly enough, decoherence has very little impact on $C P$ sensitivity when we choose model A. The analogous plot for mass ordering sensitivity is not shown because discrepancies are small and sensitivity remains well above $5 \sigma$ in any case.

\section{SUMMARY AND CONCLUSIONS}

We have tackled diverse aspects of the decoherence phenomenon in the context of neutrino oscillations. From the theoretical side, we derived a general expression for invariant decoherence matrices that are diagonal (under rotations from $\mathrm{VMB}$ to $\mathrm{MMB}$ ). The advantage of this type of matrix is its simple implementation into the matter oscillation probabilities formula as clarified in [67]. On the other hand, we have probed invariant and noninvariant decoherence matrices under realistic circumstances within a DUNE simulated environment. We have achieved a sensitivity for the decoherence parameter of $\mathcal{O}\left(10^{-24} \mathrm{GeV}\right)$ at $3 \sigma$ and for $\delta=-\pi / 2$, in the best case scenario (the invariant matrix model). A very interesting result is the presence of the degeneracy between $\Gamma$ and $\delta$, predicted at the theoretical level in [67]: depending on the value of the decoherence parameter, it becomes difficult to disentangle the scenarios where the data contain decoherence or not. Additionally, we have observed that, if decoherence plus standard oscillation is embodied within the data, the pure standard oscillation fit tends to select higher values of $\sin ^{2} \theta_{13}$ compared to the pure oscillation case. Finally, we have also seen that the decoherence phenomenon mildly affects the DUNE sensitivity on the mass hierarchy, while the $C P$ sensitivity is clearly improved but with the disadvantage that even a true lack of $C P$ violation $\left(\delta^{\text {true }}=0, \pi\right)$ can be confused with $C P$ violation.

\section{ACKNOWLEDGMENTS}

The authors acknowledge funding by the Dirección de Gestión de la Investigación at PUCP, through Grant No. DGI-2017-3-0019. They would also like to thank F. de Zela, J. Jones-Pérez, and C. Argüelles for useful discussions and a very careful reading of the manuscript.

\section{APPENDIX A: ROTATION MATRICES}

We use the following forms of the unitary operators $U_{i j}$ :

$$
\begin{aligned}
U_{23} & =\left(\begin{array}{ccc}
1 & 0 & 0 \\
0 & \cos \theta_{23} & \sin \theta_{23} \\
0 & -\sin \theta_{23} & \cos \theta_{23}
\end{array}\right) \\
U_{13} & =\left(\begin{array}{ccc}
\cos \theta_{13} & 0 & \sin \theta_{13} \\
0 & 1 & 0 \\
-\sin \theta_{13} & 0 & \cos \theta_{13}
\end{array}\right) \\
U_{12} & =\left(\begin{array}{ccc}
\cos \theta_{12} & \sin \theta_{12} & 0 \\
-\sin \theta_{12} & \cos \theta_{12} & 0 \\
0 & 0 & 1
\end{array}\right) .
\end{aligned}
$$

\section{APPENDIX B: DEFINITION OF $P, R(\delta)$ AND $\hat{\boldsymbol{R}}(\phi)$}

We define the two-dimensional rotation matrix $s(\theta)$ by

$$
s(\theta)=\left(\begin{array}{cc}
\cos \theta & \sin \theta \\
-\sin \theta & \cos \theta
\end{array}\right) .
$$

The elements of $P$ are defined as follows:

$P_{i j}\left(\delta, \phi_{1}, \phi_{2}\right)=2 \operatorname{Tr}\left[U_{T}^{\dagger}\left(\delta, \phi_{1}, \phi_{2}\right) \tau_{j} U_{T}\left(\delta, \phi_{1}, \phi_{2}\right) \tau_{i}\right]$.

Now replacing $U_{T}\left(\delta, \phi_{1}, \phi_{2}\right)=U_{\delta}^{\dagger} U_{T}(0,0,0) U_{\phi}^{\dagger}\left(\phi_{1}, \phi_{2}\right)$ in the above equation, we obtain

$$
P\left(\delta, \phi_{1}, \phi_{2}\right)=F\left(\phi_{1}, \phi_{2}\right) P(0,0,0) R(\delta),
$$

where $F, R$ are written in block-diagonal form,

$$
R(\delta)=\left(\begin{array}{llll}
\mathbb{I}_{3 \times 3} & & & \\
& s(\delta) & & \\
& & s(\delta) & \\
& & & 1
\end{array}\right)
$$

$F\left(\phi_{1}, \phi_{2}\right)=\left(\begin{array}{lllll}s\left(-\phi_{1}\right) & & & & \\ & 1 & & & \\ & & s\left(-\phi_{2}\right) & & \\ & & & s\left(\phi_{1}-\phi_{2}\right) & \\ & & & & 1\end{array}\right)$.

\section{APPENDIX C: DUNE EVENT RATES EXAMPLE}

We include the event rates for one of our simulated experiments, using the central values of the oscillation parameters and assuming $\delta=-\pi / 2$. These rates are to be 
compared directly with the oscillation probabilities in Fig. 1. While background rates are not shown explicitly, they are mostly insensitive to decoherence effects. The only exception is the $\bar{\nu}_{e}$ channel, where variations in the background become relevant since they are comparable to signal rates. It is clear that DUNE is much more sensitive to model $\mathrm{B}$ due to the large differences between $\mathrm{SO}$ rates and decoherence rates.
[1] S. Fukuda et al. (Super-Kamiokande Collaboration), Phys. Rev. Lett. 86, 5651 (2001).

[2] Q. R. Ahmad et al. (SNO Collaboration), Phys. Rev. Lett. 89, 011302 (2002).

[3] Y. Fukuda et al. (Super-Kamiokande Collaboration), Phys. Rev. Lett. 81, 1562 (1998).

[4] T. Kajita, E. Kearns, and M. Shiozawa (Super-Kamiokande Collaboration), Nucl. Phys. B908, 14 (2016).

[5] T. Araki et al. (KamLAND Collaboration), Phys. Rev. Lett. 94, 081801 (2005).

[6] F. P. An et al. (Daya Bay Collaboration), Phys. Rev. Lett. 108, 171803 (2012).

[7] P. Adamson et al. (MINOS Collaboration), Phys. Rev. D 77, 072002 (2008).

[8] J. M. Berryman, A. de Gouvêa, D. Hernandez, and R. L. N. Oliveira, Phys. Lett. B 742, 74 (2015).

[9] J. A. Frieman, H. E. Haber, and K. Freese, Phys. Lett. B 200, 115 (1988).

[10] R. Raghavan, X.-G. He, and S. Pakvasa, Phys. Rev. D 38, 1317 (1988).

[11] Z. Berezhiani, G. Fiorentini, M. Moretti, and A. Rossi, Z. Phys. C 54, 581 (1992).

[12] Z. Berezhiani, G. Fiorentini, A. Rossi, and M. Moretti, JETP Lett. 55, 151 (1992).

[13] Z. G. Berezhiani and A. Rossi, arXiv:hep-ph/9306278.

[14] V. D. Barger, J. G. Learned, P. Lipari, M. Lusignoli, S. Pakvasa, and T. J. Weiler, Phys. Lett. B 462, 109 (1999).

[15] J. F. Beacom and N. F. Bell, Phys. Rev. D 65, 113009 (2002).

[16] A. S. Joshipura, E. Masso, and S. Mohanty, Phys. Rev. D 66 , 113008 (2002).

[17] A. Bandyopadhyay, S. Choubey, and S. Goswami, Phys. Lett. B 555, 33 (2003).

[18] S. Ando, Phys. Rev. D 70, 033004 (2004).

[19] G. Fogli, E. Lisi, A. Mirizzi, and D. Montanino, Phys. Rev. D 70, 013001 (2004).

[20] S. Palomares-Ruiz, S. Pascoli, and T. Schwetz, J. High Energy Phys. 09 (2005) 048.

[21] M. C. Gonzalez-Garcia and M. Maltoni, Phys. Lett. B 663, 405 (2008).

[22] M. Maltoni and W. Winter, J. High Energy Phys. 07 (2008) 064.

[23] P. Baerwald, M. Bustamante, and W. Winter, J. Cosmol. Aatropart. Phys. 10 (2012) 020.

[24] D. Meloni and T. Ohlsson, Phys. Rev. D 75, 125017 (2007).

[25] C. R. Das and J. Pulido, Phys. Rev. D 83, 053009 (2011).

[26] L. Dorame, O. G. Miranda, and J. W. F. Valle, Front. Phys. 1, 25 (2013).
[27] R. A. Gomes, A. L. G. Gomes, and O. L. G. Peres, Phys. Lett. B 740, 345 (2015).

[28] R. Picoreti, M. M. Guzzo, P. C. de Holanda, and R. L. N. Oliveira, Phys. Lett. B 761, 70 (2016).

[29] T. Abrahão, H. Minakata, and A. A. Quiroga, J. High Energy Phys. 11 (2015) 001.

[30] M. Bustamante, J. F. Beacom, and K. Murase, Phys. Rev. D 95, 063013 (2017).

[31] A. M. Gago, R. A. Gomes, A. L. G. Gomes, J. Jones-Perez, and O. L. G. Peres, J. High Energy Phys. 11 (2017) 022.

[32] P. Coloma and O. L. G. Peres, arXiv:1705.03599.

[33] M. V. Ascencio-Sosa, A. M. Calatayud-Cadenillas, A. M. Gago, and J. Jones-Prez, Eur. Phys. J. C 78, 809 (2018).

[34] M. C. Gonzales-Garcia, M. M. Guzzo, P. Krastev, and H. Nunokawa, Phys. Rev. Lett. 82, 3202 (1999).

[35] S. Bergmann, M. M. Guzzo, P. C. de Holanda, P. Krastev, and H. Nunokawa, Phys. Rev. D 62, 073001 (2000).

[36] M. M. Guzzo, P. C. de Holanda, and O. L. G. Peres, Phys. Lett. B 591, 1 (2004).

[37] A. M. Gago, M. M. Guzzo, H. Nunokawa, W. J. C. Teves, and R. Z. Funchal, Phys. Rev. D 64, 073003 (2001).

[38] A. M. Gago, M. M. Guzzo, P. C. de Holanda, H. Nunokawa, O. L. G. Peres, V. Pleitez, and R. Z. Funchal, Phys. Rev. D 65, 073012 (2002).

[39] T. Ohlsson, Rep. Prog. Phys. 76, 044201 (2013).

[40] A. Esmaili and A. Y. Smirnov, J. High Energy Phys. 06 (2013) 026.

[41] D. Colladay and V. A. Kostelecky, Phys. Rev. D 55, 6760 (1997).

[42] S. R. Coleman and S. L. Glashow, Phys. Rev. D 59, 116008 (1999).

[43] S. R. Coleman and S. L. Glashow, Phys. Lett. B 405, 249 (1997).

[44] D. Colladay and V. A. Kostelecky, Phys. Rev. D 58, 116002 (1998).

[45] P. Adamson et al. (MINOS Collaboration), Phys. Rev. Lett. 105, 151601 (2010).

[46] A. A. Aguilar-Arevalo et al. (MiniBooNE Collaboration), Phys. Lett. B 718, 1303 (2013).

[47] Y.F. Li and Z.h. Zhao, Phys. Rev. D 90, 113014 (2014).

[48] J. Ellis, N. E. Mavromatos, and D. V. Nanopoulos, Phys. Lett. B 293, 37 (1992); Int. J. Mod. Phys. A 11, 1489 (1996).

[49] F. Benatti and R. Floreanini, Ann. Phys. (N.Y.) 273, 58 (1999).

[50] S. Hawking, Commun. Math. Phys. 87, 395 (1982); Phys. Rev. D 37, 904 (1988); 53, 3099 (1996); S. Hawking and C. Hunter, Phys. Rev. D 59, 044025 (1999). 
[51] F. Benatti and R. Floreanini, J. High Energy Phys. 02 (2000) 032.

[52] F. Benatti and R. Floreanini, Phys. Rev. D 64, 085015 (2001).

[53] A. M. Gago, E. M. Santos, W. J. C. Teves, and R. Z. Funchal, arXiv:hep-ph/0208166.

[54] R. L. N. Oliveira and M. M. Guzzo, Eur. Phys. J. C 69, 493 (2010).

[55] R. L. N. Oliveira and M. M. Guzzo, Eur. Phys. J. C 73, 2434 (2013).

[56] R. L. N. Oliveira, Eur. Phys. J. C 76, 417 (2016).

[57] E. Lisi, A. Marrone, and D. Montanino, Phys. Rev. Lett. 85, 1166 (2000).

[58] Y. Farzan, T. Schwetz, and A. Y. Smirnov, J. High Energy Phys. 07 (2008) 067.

[59] G. Barenboim and N. E. Mavromatos, J. High Energy Phys. 01 (2005) 034.

[60] P. Bakhti, Y. Farzan, and T. Schwetz, J. High Energy Phys. 05 (2015) 007.

[61] A. M. Gago, E. M. Santos, W. J. C. Teves, and R. Z. Funchal, Phys. Rev. D 63, 073001 (2001).

[62] D. Morgan, E. Winstanley, J. Brunner, and L. F. Thompson, Astropart. Phys. 25, 311 (2006).

[63] G. L. Fogli, E. Lisi, A. Marrone, D. Montanino, and A. Palazzo, Phys. Rev. D 76, 033006 (2007).

[64] R. L. N. Oliveira, M. M. Guzzo, and P. C. de Holanda, Phys. Rev. D 89, 053002 (2014).

[65] G. B. Gomes, M. M. Guzzo, P. C. de Holanda, and R. L. N. Oliveira, Phys. Rev. D 95, 113005 (2017).

[66] M. M. Guzzo, P. C. de Holanda, and R. L. N. Oliveira, Nucl. Phys. B908, 408 (2016).
[67] J. A. Carpio, E. Massoni, and A. M. Gago, Phys. Rev. D 97, 115017 (2018).

[68] G. B. Gomes, D. V. Forero, M. M. Guzzo, P. C. De Holanda, and R. L. N. Oliveira, arXiv:1805.09818.

[69] P. Coloma, J. Lopez-Pavon, I. Martinez-Soler, and H. Nunokawa, Eur. Phys. J. C 78, 614 (2018).

[70] R. Acciarri et al. (DUNE Collaboration), arXiv:1512.06148.

[71] S. Hernández, Magister tesis, Pontificia Universidad Católica del Perú, 2016.

[72] V. Gorini, A. Frigerio, M. Verri, A. Kossakowski, and E. C. G. Sudarshan, Rep. Math. Phys. 13, 149 (1978). High Energy Physics-Phenomenology.

[73] F. Benatti and R. Floreanini, Phys. Rev. D 64, 085015 (2001).

[74] G. Lindblad, Commun. Math. Phys. 48, 119 (1976).

[75] R. Alicki and K. Lendi, Quantum Dynamical Semigroups and Applications, Lect. Notes Phys. Vol. 286 (SpringerVerlag, Berlin, 1987).

[76] T. Alion et al., arXiv:1606.09550.

[77] I. Esteban, M. C. Gonzalez-Garcia, M. Maltoni, I. MartinezSolar, and T. Schwertz, J. High Energy Phys. 01 (2017) 087.

[78] www.nu-fit.org.

[79] P. Huber, M. Lindner, and W. Winter, Comput. Phys. Commun. 167, 195 (2005).

[80] P. Huber, J. Kopp, M. Lindner, M. Rolinec, and W. Winter, Comput. Phys. Commun. 177, 432 (2007).

[81] C. A. A. Delgado, J. Salvado, and C. N. Weaver, arXiv: 1412.3832 .

Correction: The previously published Figs. 2 and 3 and related text in the first sentence of the second paragraph in Sec. III B contained errors and have been fixed. 